\title{
Geschichte, Geschichten und Mythen in Dea Lohers Olgas Raum
}

\author{
[History, stories and myths in Dea Lohers Olga's Room] \\ http://dx.doi.org/10.11606/1982-8837192937
}

\begin{abstract}
Alexandra Ludewis ${ }^{1}$
Abstract: Over the past few decades, Germany's division and subsequent unification have altered he way the country's history is understood, with Eastern and Western narratives needing to merge into a new master narrative. The different accounts of the life and death of the JewishGerman communist Olga Benario, partner of the Brazilian freedom fighter, Luis Carlos Prestes, serve as a powerful case study for Germany's author Dea Loher. In her theatre play Olga's Room (1994) the empty stage walls are used in such a way as to serve as a screen for history, her stories and myths, before the actors deconstruct these walls themselves by disclosing their own tragic flaws. In the process, the construction mechanisms that lie behind the history and stories are revealed. The audience is thereby pushed into an active reception role, not least when left with the empty space which Olga and the other actors leave one by one. Escapism into a myth is no longer possible; after all, the realisation that violence and general fallibility are profoundly human prevents this folly.
\end{abstract}

Keywords: Dea Loher; historical Drama; political Drama; Mythos; History

Zusammenfassung: In den letzten Jahrzehnten haben Deutschlands Teilung und die darauf folgende Wiedervereinigung die Art und Weise beeinflusst, wie die Geschichte des Landes verstanden wird, wobei östlich und westlich geprägte Erzählungen zu einer neuen Geschichtsschreibung verschmolzen. Die verschiedenen Sichtweisen auf das Leben und den Tod der jüdisch-deutschen Kommunistin Olga Benario, der Lebensgefährtin des brasilianischen Freiheitskämpfers Luis Carlos Prestes, dienen der deutschen Autorin Dea Loher in diesem Zusammenhang als aussagekräftige Fallstudie. In ihrem Theaterstück Olgas Raum (1994) werden die leeren Wände der Bühne genutzt, um auf ihnen Geschichte, Geschichten und Mythen zu projizieren, bevor die Figuren durch ihre eigenen tragischen Fehler diese Entwürfe dekonstruieren. Dabei werden die Konstruktionsmechanismen, die hinter der Entstehung von Geschichte und Geschichten liegen, enthüllt. Das Publikum wird so in eine aktive Rezipientenrolle gedrängt, nicht zuletzt wenn es mit dem leeren Raum, den Olga und die anderen Akteure nach einander verlassen, alleingelassen wird. Eine Flucht in den Mythos ist nicht mehr möglich in Anbetracht der Erkenntnis, dass Gewalt und allgemeine Fehlbarkeit zutiefst menschlich sind.

Stichwörter: Dea Loher; historisches Drama; politisches Theater; Mythos; Geschichte

\footnotetext{
${ }^{1}$ The University of Western Australia, German Studies, 204/35, 6009, Stirling Hwy Perth, Perth, Australia.E-mail: alexandra.ludewig@uwa.edu.au
} 


\section{Einleitung}

1990 fand in Deutschland mit der historischen Zäsur der Währungsunion und der Wiedervereinigung ein ideologischer Abschied vom Kommunismus statt. Somit wurde intellektuell der Boden bereitet für eine Neubestimmung der Geschichtsschreibung, die aus dem Schatten des Dritten Reiches heraus zu schreiten schien. In der Euphorie über den Mauerfall sahen viele Historiker eine neue Ära eingeläutet, in welcher man seine Perspektive für die Zukunft statt von dem Erbe vor 1945 nun von den Ereignissen seit 1989 bestimmt sehen wollte. Angesichts dieses neuen Deutschlands setzte eine Neuschreibung der Geschichte ein. JUDT spricht in diesem Zusammenhang von einer „psychologisch und politisch willkommenen Allianz zwischen historischem Neuanfang und kollektiver Amnesie“ (1998: 7f.). Andere sahen sogar ein Ende der Geschichte nahen (FUKUYAMA 1989: 3). Diese Jahre fielen zusammen mit den literarischen Anfängen der Dramatikerin Dea Loher. ${ }^{2}$ Frisch graduiert von der LMU-München, hatte sich Loher nach Südamerika verabschiedet und von dort die monumentale Verschiebung der geo-politischen Lage in Europa verfolgen können. In dieser Phase der Euphorie über Gegenwärtiges und Zukünftiges besann sich Loher in ihrem Bühnenerstling auf Vergangenes. Sie wandte sich bewusst einem Stoff zu, der nicht nur ihre alte Heimat und deren schwere Geschichte mit ihrem Interesse für Brasilien verband, sondern auch eine Gratwanderung zwischen Weltentwürfen und Lebenskonzepten beinhaltete. Sie spürte in ihren Recherchen zu Olgas Raum (1992) dem Lebensweg von Olga Benario (1908-1942) nach, einer militanten jüdischdeutschen Kommunistin, die als Lebensgefährtin des brasilianischen Revolutionärs Luis Carlos Prestes von der brasilianischen Regierung hochschwanger an das Dritte Reich ausgeliefert und dort nach langer Inhaftierung vergast wurde. Die Hinwendung zum Holocaust kommentiert die Autorin selbst als ,die Verantwortung, die die Geschichte dieses Landes mit sich bringt.“ (LOHER in ŠTĚDROŇ 2004: 266).

Die Zuschreibungen ,jüdisch“, „militant“, „deutsch“ und „kommunistisch“ werden in Lohers Text genauso hinterfragt, wie die Kategorien von Täter und Opfer.

\footnotetext{
${ }^{2}$ Auch in Brasilien ist Loher (1964- ) keine Unbekannte: „Im Rahmen des Festivals Territorio livre in São Paulo fanden Lohers erstes Drama Olgas Raum (1990) sowie eines ihrer neueren Stücke Das Leben auf der Praça Roosevelt (2004) großen Anklang (Regie: Andreas Kriegenburg). Dies mag zum einen daran liegen, dass beide Stücke in Brasilien spielen, zum anderen darin begründet sein, dass beide Dramen einen sozialkritischen Inhalt transportieren.” (HAAS 2007: 269). Siehe auch MIGUEL (2016).
} 
Die Aktion auf der Bühne wird genutzt, um Schwarz-Weiß-Zuschreibungen zu verkomplizieren und Vergangenheitsaufarbeitung zu betreiben, die nicht als Geschichte der Sieger gereicht. Lohers Annäherung an den Stoff erfolgte dabei in mehreren Phasen, die kontextgebunden, entweder eine südamerikanische, eine ostdeutsche oder eine westdeutsche Perspektive auf die Figuren hervorheben. Olgas Raum thematisiert mit dem auf der Bühne entworfenen Raum und seinen kahlen Wänden genau diese Projektionsflächen, auf denen mehrere Versionen von Olga vor den Augen der Zuschauer entstehen. Die eine wahre Geschichte der Olga Benario Prestes gibt es nicht. So wird auch auf der Bühne immer wieder eine gewisse Beliebigkeit der Entwürfe in den Vordergrund gerückt. ${ }^{3}$ Jede Annäherung an ihre Geschichte entpuppt sich als eine subjektive Interpretation der jeweils erzählenden Person.

Bewusst belebte Dea Loher zu dieser Zeit des scheinbaren Triumphes des Westens über den Osten mit Olga Benario eine Legionsfigur der Sozialisten, die nach 1945 gerade im Osten zur Ikone stilisiert worden war. Anna Seghers schrieb für den Internationalen Tag der Frau von 1951 einen biographischen Sketch, der idealtypisch das Eigene und Fremde verklärte. Stephan Hermlin erzählte in seinem Aufsatz aus dem Jahre 1951 die Geschichte der Olga Benario in der Diktion der Zeit des Stalinismus und der uneingeschränkten Verehrung der Sowjetunion. Die Vita dieser „Heldin einer großen Liebe" gereichte ihm zur „Legende einer Heiligen unserer Zeit“, propagandistisch zum ,große[n] Epos von der Brüderlichkeit der Völker und [...] blutige[n] Farce der Solidarität der Henker.“ (HERMLIN 1951: 64-66) Schon bald zierte Olga Benario Prestes' Portrait eine 20-Pfennig-Briefmarke der DDR. Ruth Werner trug 1961, im Jahr des Mauerbaus, mit einer Huldigung von Olga Benarios „tapferem Leben“" weiter zur Gedenkkultur bei. ${ }^{4}$

Lohers Theatertext versuchte somit um 1990 mit ihrer Wahl der Protagonistin dem westlich-kapitalistischen Teil Deutschlands eine Figur nahezubringen, die in deren

\footnotetext{
${ }^{3}$ Diese Aussage von Loher missverstehend, inszenierte die Studiobühne Köln unter der Regie von Inka Neubert 1998 das Stück unter dem Titel „Olga Benario“ nach Dea Loher auf. Im Stück heißt es dagegen: „Für jede Geschichte gibt es eine andere. Die Wahrheit wird ständig sabotiert durch die Phantasie, durch die Einbildungskraft. Die Wahrheit ist die Einbildungskraft.“ (LOHER 1994: 21)

${ }^{4}$ In der DDR wurden zudem etliche Straßen, sowie Dutzende von Fabriken, Schulen und Brigaden nach ihr benannt. Trotz einer Welle der Umbenennungen nach dem Mauerfall ist Olga Benario auch im wiedervereinigten Deutschland noch Namensgeberin für einige Straßen, Galerien und (Jugend-) Klubs, Jugendherbergen, gemeinnützige Einrichtungen und Schulen geblieben.
} 
Wirklichkeit bis dahin noch keinen großen Nachhall gefunden hatte. ${ }^{5}$ Loher konzentriert sich in ihrem Stück jedoch nicht nur auf die „Fama“ sondern auch auf die „Hamartia“, auf menschliche Schwäche. Ihr Stück zerfällt nicht zuletzt deswegen regelrecht in zwei Teile, einen Rahmen, der Einleitung und Ende bildet, und der sich in faktisch-neutralem Stil stark an den biographischen Vorgaben orientiert, sowie einen Hauptteil, in welchem Loher stark fiktionalisiert Olgas Zeit im brasilianischen Gefängnis dramatisiert.

Dabei bedient sich Loher einer lyrisch-erzählenden Struktur, sodass das Stück selbst handlungsarm erscheint. Zumeist wird auf der Bühne Vergangenheit verhandelt bzw. besprochen, und selbst die Dialogpassagen sind in weiten Teilen episch angelegt. Der größte Sprechanteil entfällt auf Olga, die in langen Monologpassagen ihre Lebensgeschichte in ihrer Gefängniszelle Revue passieren lässt, wie um sich selbst ihrer Person zu vergewissern. Zudem teilt sie sich ihren Mitgefangenen Ana Libre und Genny mit, wobei die Dialoge - den Monologen nicht unähnlich - eine Spiegelfunktion erfüllen. Es geht weiterhin nur um Olga und ihre Person, die bisweilen im Zerrspiegel oder in einer weniger beschönigenden oder entfremden Reflektion erscheint. Olgas Raum oder Zelle wird ihr in den PAS DE DIABLE-Passagen von ihrem Folterer Filinto Müller streitig gemacht. Sie lernt jedoch aus den Verhören und Gewaltexzessen ihres Peinigers und versteht es schlussendlich, die Rollen in einem psychologischen Spiel zu vertauschen. Wie Loher betont: „Es geht in dem Stück darum, ob und wie Gewalt legitimiert werden kann, von staatlicher Seite einerseits, und als Mittel des Widerstandes andererseits.“(LOHER in ŠTĚDROŇ 2004: 267).

Statt die historische Figur zu rehabilitieren oder glorifizieren, die in der Geschichtsschreibung der DDR für Vereinnahmung und in der BRD für einen blinden Fleck steht, geht es Loher um Differenzierung. Der Skizzierung des historischen Rahmens in ihrem Stück stehen somit die Rekonstruktion und Dekonstruktion von Geschichte(n) gegenüber, in denen Loher Aspekte der verbalen Identitätsvergewisserung behandelt sowie Überlegungen zur Fehlbarkeit von Menschen anstellt. In ihrem Theatertext orientiert sich Loher einerseits stark an den biographischen Stationen, um die Ausgangssituation von Olga im brasilianischen

\footnotetext{
${ }^{5}$ Erst im Gefolge von Dea Loher setzte eine Renaissance von Olga Benario ein, in Deutschland aber auch international. So wurde für sie in den 1990er Jahren ein Stoplerstein eingesetzt (vor ihrem Wohnhaus Innstr. 24 / Ecke Donaustraße in Berlin-Neukölln). Unter der Regie von Sergio Toledo wurde ihr ein Film gewidmet. Panitz publizierte eine Biographie zu Jorge Amados Leben, in welcher Olga Benario und Luis Prestes ausführliche Erwähnung finden. Robert Cohens Exil der frechen Frauen verehrte Olga 2006 eine Hauptrolle. Auch in der brasilianischen Erinnerungskultur hat Olga ihren Platz (vgl. MORAIS 1998).
} 
Gefängnis realitätsgetreu zu skizzieren, bevor sie einerseits jede Lebenserzählung als Mythosbildung dekonstruiert; andererseits nimmt sie Bezug auf anthropologische Konstanten - der menschlichen Fehlbarkeit. Deshalb soll Loher im Nachfolgenden methodologisch gefolgt werden, d. h., der Dekonstruktion die Rekonstruktion vorangehen, um Olgas Lebenserzählung in ihrer Entstehungsgeschichte, ihren soziokulturellen wie biographischen Voraussetzungen zu verorten.

\section{Die eine Geschichte der Olga Benario in der Rahmenrekonstruktion}

Olga Benario wurde am 12. Februar 1908 in München in eine bürgerliche, assimilierte jüdisch-deutsche Anwaltsfamilie hinein geboren. Bereits als Jugendliche wurde sie aktenkundig und in den Polizeiakten der Weimarer Republik als kommunistische Agitatorin tituliert. Als 17-Jährige zog sie nach Berlin-Neukölln, wo sie sich dem Kommunistischen Jugendverband anschloss. Im Rahmen dieser Jugendorganisation fand Olga ihre erste große Liebe. Loher folgt in ihrem Stück ${ }^{6}$ in emotions- und schmuckloser Sprache den biographischen Daten und Aufenthaltsorten von Olga Benario: „OLGA Da ist der Genosse Otto Braun, mit dem ich von München nach Berlin zog, um im Untergrund zu arbeiten für die KP. Da war ich 17. Und er mein Geliebter.“ (LOHER 1994: 6) Olga und ihr Freund wurden verhaftet:

,Landesverrat' hieß es für Otto Braun. Ich sollte Komplizin sein, bei der Kampagne zur Enteignung der Fürsten. Sie konnten nichts beweisen. Zwei Monate saß ich in Moabit. Achtzehn Monate wartet Otto Braun auf den Prozeß. Wir haben ihn befreit. Zu sechst. Aus dem Gerichtssaal. [...] Sie suchten uns. Wir flohen mit gefälschtem Paß nach Rußland. (LOHER 1994: 7)

In Moskau wurde Olga als Delegierte des V. Weltkongresses der Kommunistischen Jugendinternationale gefördert. „OLGA Ich lernte Russisch. Stand in vielen Sälen und hielt Reden. Wurde ins Zentrale Komitee gewählt. [...] Otto Braun ging fort. Eine andere Frau. Die hatte Zeit für ihn.“ (LOHER 1994: 7) Eine neue Mission bringt einen weiteren Ortswechsel mit sich: „OLGA Der Auftrag: Ich sollte Luis Carlos Prestes’ persönliche Begleitung sein, seine Sicherheit verbürgen.“ (LOHER 1994: 7) Das war 1934. Olga Benario ging von Moskau aus mit dem Brasilianer Luis Carlos Prestes

\footnotetext{
${ }^{6}$ Im folgenden beziehen sich alle in eckigen Klammern stehenden Seitenangaben auf die Textausgabe des Verlags der Autoren von 1994.
} 
(1899-1990) nach Rio de Janeiro, wo sich beide an revolutionären Aufständen beteiligten. Stakkatohaft, in gehetzter Rede, jedoch im Präsens ohne jedes epische Präteritum, ohne jede Verklärung und jedes überflüssige Wort beschreibt Olga in Lohers Stück die Ereignisse in Südamerika:

Die Linken gründen eine Allianz zur Nationalen Befreiung. Befreiung von der Diktatur. Prestes übernimmt den Vorsitz. 35 Rio de Janeiro: Die Regierung verbietet die Nationale Befreiung. Unruhen. Wir rufen die Revolution aus. Die Regierung ruft den Notstand aus. Und mobilisiert ihre Streitkräfte. Die Revolution bricht so schnell zusammen wie sie ausgebrochen ist. Sie wird niedergeknüppelt. Massenverhaftungen. (LOHER 1994: 9)

Als habe sie in diesem Strudel der Ereignisse die Kontrolle verloren, so erscheint die unausbleibliche Folge, als sie und Prestes 1936 von der brasilianischen Polizei und der deutschen Gestapo verhaftet werden: „Sie verhören. Prestes wird verurteilt werden, erst zu 17, dann noch einmal zu 30 Jahren Zuchthaus, macht 47 Jahre.“ (LoHER 1994: 9) Als Deutsche bleibt Olga Benario ein anderes Schicksal vorbehalten. Die Gestapo, die Präsident Getúlio Vargas” „Estado Novo“ (portugiesisch: „Neuer Staat“) unterstützte und der brasilianischen Regierung bereits bei der Verfolgung und Festnahme der linken Revolutionäre behilflich war, plädiert für eine Auslieferung Benarios. Trotz internationaler Proteste wurde die hochschwangere Olga Benario im September 1936 von den brasilianischen Behörden an die Gestapo ausgeliefert. Im deutschen Frauengefängnis gebar sie am 27. November 1936 ihre Tochter Anita Leocadia, von der sie jedoch Anfang 1938 getrennt wurde. Diese Ereignisse kommentiert Olga in Dea Lohers Theaterstück mit mehr Emotionen:

OLGA Ich hatte eine Tochter Anita und war im Gefängnis. Ich durfte sie in meiner Zelle behalten, solange ich stillen konnte. Das waren vierzehn Monate. [...] Anita ist fort. Mein Kind ist fort. Sie haben sie fortgenommen, und mich ins Lager gebracht. [Sie] ist [...] bei Verwandten in Paris. Oder Mexiko? Ich weiß es nicht. (LOHER 1994: 33)

Von ihrer Tochter getrennt, ohne ihren Partner und ohne jede Aussicht auf Besserung, sieht Olga ihrem Ende entgegen. Sie wird nach Lichtenburg gebracht. Von 1933 bis 1939 befand sich hier eines der vielen Konzentrationslager für politische Häftlinge. Von dort wurde sie 1939 in das Konzentrationslager Ravensbrück überführt. Dea Loher lässt Olga ihre Gefängnisjahre wie folgt summieren: „Das ist mein Raum. Eine Zelle im KZ Ravenbrück. Wie lange bin ich hier? [...] 42414039 KZ Ravensbrück. 3938 KZ Lichtenburg. 383736 Frauengefängnis Berlin. 36 Gefängnis in Rio de Janeiro, 
Brasilien.“ (LOHER 1994: 6) Die Jahre werden für Olga Benario nur durch die Ortswechsel unterbrochen. Am Ende dieser unfreiwilligen Odyssee durch verschiedene Gefängnisse landet Olga Benario in der „Heil- und Pflegeanstalt“ Bernburg, wo sie 1942 mit Zyklon B ermordet wurde.

\section{Olgas Raumentfaltung bei Dea Loher}

Die Mythosbildung, die besonders Hermlin in seinem Aufsatz vornahm, steht in starkem Kontrast zu der Behandlung, die Olgas Biographie in Dea Lohers Stück erfährt. Gemäß ihrem Motto, „Kunst müsse immer in irgendeiner Weise Realität infrage stellen oder neue Realität sichtbar machen“ (MÜLLER in FISCHBORN 1981: 43), arbeitet Loher nicht am Mythos, aber auch nicht an einer faktengetreuen Geschichtsschreibung. Sie sucht den Freiraum dahinter und arbeitet an einer dritten Version. Besonders inspiriert scheint Dea Loher deshalb von den Leerstellen und Auslassungen in Hermlins Legendenerzählung. HERMLIN deutet z.B. an, dass sich in ruhigen Stunden manchmal die Mädchen um Olga Benario scharten, um von ihrem Leben zu hören und dem von Luis Carlos Prestes, dem Ritter der Hoffnung (1951: 65). Bei Dea Loher erzählt Olga ebenfalls davon und doch erzählt sie dadurch noch vielmehr. Das Prinzip des Geschichtenerzählens an sich und der Mythosbildung per se, das Olga ebenso wie Loher in dem Fall gemein haben, wird nun von Loher in Olgas Raum vorexerziert. Der entstehende Raum aus Wort und Schall wird zur Kulisse für Lohers Problematisierung von Realität. Olgas Erzählungen zeugen von der Möglichkeit freier Selbstsetzung durch Sprechen und verdeutlichen, wie Sprache zu Macht und Ohnmacht eines jeden beiträgt.

Sprache im Allgemeinen, inklusive aller Formen der Körpersprache, wie Mimik, Gestik, Habitus usw. ist ein Medium der Kommunikation sowie als deren Bestandteil ein Mittel der Selbstvergewisserung und fungiert als Sinnstiftungsagent. Die verbalen und nonverbalen Ausdrucksvarianten orientieren sich dabei primär am Funktionsrahmen, wobei trotz der visuellen Variante in der Vorführungspraxis von Olgas Raum im Folgenden nur auf die verbale Kommunikation eingegangen werden soll. Die Analyse des Theatertextes im Gegensatz zu den vielfältigen visuellen Interpretationen der Inszenierungen empfiehlt sich nicht zuletzt, da Dea Loher mit Regieanweisungen äußerst sparsam umgeht und damit das Augenmerk auf die sprachliche Diktion lenkt. 
In Olgas Raum wird Sprechen als exemplarisches Medium des Entwurfs und des experimentellen Durchspielens verschiedenster Inszenierungen von Subjektivität genutzt. Dabei ist das Subjekt nichts ursprünglich Existierendes, das sich verbal zum Ausdruck bringt, vielmehr konstituiert es sich im sprachlichen Geschehen immer wieder neu, nimmt im Sprechen Gestalt an und verobjektiviert sich, fungiert als solches als ständige Frage nach einer Antwort. Somit wird das Erzählen zur Produktivität, die aus sich selbst die Erfindung und Ausdifferenzierung von Subjektivität hervortreibt. Praktisch stehen dabei Selbstvergewisserung und Selbstentwurf in einem permanenten Spannungsverhältnis im phasenweise erprobten Prozess von Findung und Erfindung. Olgas Autosuggestion ist eine Manifestation ihrer versuchten Selbstvergewisserung, die ihr gerade in der Situation der Bedrohung ihres Daseins zu einer seelischen Plombe gereicht. Den gleichen Zweck erfüllen generell Erzählungen verschiedenster Ausprägungen, z. B. Märchen, religiöse Texte oder Mythen. Sie fungieren wie eine Utopie, d. h., als Gegenentwurf oder Projektion einer perfekten Weltordnung, in welcher das Individuum sich aufgehoben fühlt. In diesem Prozess der Selbsterfindung spielen die vielfältigen Formen und Aspekte des Vergessens in ebenso starkem Maße eine Rolle wie das Erinnern. In Olgas Situation ist die selektive Erinnerung das Vehikel der Flucht aus der Inhaftierung: „Nur wenn ich mich ganz genau erinnere, werde ich die Zukunft erleben.“ (LOHER 1994: 6) Diese Erinnerungsarbeit vollzieht sich über - in Worten gemalte - Bilder:

Haft. Graue Wände. Ich spreche mit mir selbst, um nicht verrückt zu werden. Ich zeige mir Bilder, erzähle mir Geschichten. Hier im Kopf sitzt mein Erinnerungsalbum. Sich jeden Tag an ein Ereignis erinnern und es genau rekonstruieren. (LOHER 1994: 6)

Olga benutzt ihre Erfahrungen als Steinbruch für die nachträgliche Entdeckung, Situierung und Bestimmung der eigenen Identität, es ist emotionale Schwerstarbeit, die sich selbst sehr vorsichtig vollzieht. In kleinen Schritten reflektiert sie in ihren Erinnerungen ihr Gewordensein und benennt ihre verschiedenen Stationen und Rollen: Begleiterin, Assistentin, Freundin, Sekretärin, Geliebte, Mutter, Märtyrerin. Der Weg zu dieser Erkenntnis wird sehr behutsam beschritten. Wie in einem Stationendrama lässt Olga in der Enge der Zelle ihr Leben Revue passieren, erinnert sich ihrer Vita, indem sie sich реи а реи an die größeren Zusammenhänge heran arbeitet: „Ich bin Olga. Das ist mein Raum. Eine Zelle im KZ Ravensbrück. [...] ich, Olga [...] Be-na-rio. Bertha Emil Nordpol Anton Richard Ida Otto. Jahrgang 1908.“ (LOHER 1994: 6) Die 
identitätsbestimmenden, von Behörden immer vermerkten Kategorien, Name, Wohnort, Staatsangehörigkeit, geht Olga systematisch durch: „Staatsbürgerschaft —: Jüdin. — Staatsbürgerschaft —: deutsch. —“ (LOHER 1994: 6) Die lebensrettende „Staatsbürgerschaft: brasilianisch“, die ihr nach der Geburt des Kindes zugestanden hätte, bleibt ihr jedoch verwehrt.

Olgas nüchternen Worte zur eigenen Person stehen im Kontrast zu der Überhöhung Luis Carlos Prestes’: „Brasilianer. Ritter der Hoffnung. Anführer des bewaffneten Widerstandes in seinem Land. Gegen die Diktatur.“ (LOHER 1994: 6) Prestes wird mit Konnotationen eines Heilsbringers belegt, wobei die Attraktivität des Mannes und Olgas Gefolgschaft nicht ohne ironische Distanz vermerkt werden:

OLGA [...] der [Hauptmann aus Bolivien] erzählte von dem Hauptmann aus Brasilien so: ER hat die Stärke einer Python, [...] dabei mutig wie der gefleckte Jaguar, die onça pintada, und flink wie das tatú, das Gürteltier, zugleich gerissener Stratege, doch vor dem Kampf, wenn jeder Schritt des Kampfes festgelegt, ein Heiliger [...] So sagte er. Sollte ich die Volksgeschichten glauben, ganz Südamerika e i n Tonfall der Bewunderung — die Zeit der Helden lang vorbei, der Bolivianer wollte gern Geschichte schreiben. (LOHER 1994: 8)

Olga wird als Luis Carlos Prestes’ persönliche Begleitung abgestellt: „Ich hatte einen Parteiauftrag zu erfüllen, der sich zufällig mit meiner Überzeugung deckte und auch mit meiner menschlichen Neigung nicht völlig unvereinbar war.“ (LOHER 1994: 24) Somit kann Olga ihren Werdegang rückblickend mit gutem Gewissen als den richtigen bezeichnen und sich selbstbewusst zu ihrem Werden und Sein bekennen. Dieses Annehmen, dieses Akzeptieren der eigenen Person bildet den Kern des Dramas und hinterlässt beim Betrachter den Eindruck von einer starken, reflektierten Frau. Obwohl die Rahmenstruktur bzw. Kreisstruktur des Dramas einerseits Olgas ausweglose Situation in den Fängen der Justiz betont, signalisiert sie andererseits Olgas stoische Selbstsetzung: „Ich bin Olga. [...] Das ist mein leerer Raum.“ (LOHER 1994: 34) Diesen Raum füllt sie mit ihren Erzählungen. Dabei vereinigen sich Familien- und Privatgeschichte sowie Weltgeschichte, verbal, in Kommunikation mit anderen sowie im Selbstgespräch, zur Stütze des eigenen Denkens, als Ausdruck für tiefe Empfindungen und als ästhetischer Akt. Innerhalb dieses Funktionsspektrums setzt Olga die Sprache, ob im Monolog oder Dialog, zu verschiedenen Zwecken ein. Sie dient der Unterhaltung, dem Ausdruck von Herrschaftsansprüchen, der Kooperation, der Manipulation, Beruhigung, Belehrung und Täuschung. Dadurch wird Sprache per se zum Gegenstand der Untersuchung, zum Objekt der Faszination für die Geschichten 
erzählende Olga und die Geschichtenerzählerin Loher zugleich. Loher betont deshalb auch besonders die Funktion von Sprache für die Traditionsvermittlung in Form von Märchen, Mythen, Sagen oder auch die Kundgabe-, Auslösungs- und Darstellungsfunktion von Sprache. Sprechen als Vehikel sozialer Identität reflektiert und zementiert soziale Strukturen, und fungiert als wichtiges Medium politischer Macht, was Loher in Olgas Raum exemplarisch durchexerziert. Zum einen bietet verbalisierte Erinnerung für Olga einen Selbstschutz, aber zum anderen auch Trost für Mitgefangene: „GENNY [...] Erzähle, Olga, gib mir einen Talisman aus Worten, den mir niemand nehmen kann. Worte, unter denen ich mich verstecken kann wie unter einem weiten Mantel, wie in einem Wald.“ (LOHER 1994: 7) Darauf entgegnet „OLGA [...] Ich erzähle dir Geschichten. [...] GENNY Wie eine Mutter. Nachts, bei Gewitter, um die Angst zu vertreiben.“(LOHER 1994: 25)

Dieser positiven, konstruktiven Funktion von Geschichte(n) steht das Potenzial ihrer negativen Aspekte gegenüber, denn Olga setzt Sprache immer wieder zu Zwecken der Maskerade und Täuschung ein: „OLGA [...] Kein Ort sicher, keine Zeit ruhig, kein Gedanke zu Ende - Flucht, Verstecken, Tarnen. — Alle Tage anders. — Gleich bleibt nur das Täuschen.“ (LOHER 1994: 8) Dieser Umstand verstärkt sich, als Olga in Gefangenschaft gerät. „OLGA [...] Ich will ihn [meinen Verhörer] täuschen so lange wie möglich. Ihn mit vielen Geschichten verwirren und mich nicht preisgeben.“ (LOHER 1994: 9) Durch die anvisierte Manipulation mit Hilfe von Worten soll die Sprache als Instrument zur Verfolgung bestimmter Zwecke im Gegensatz zu ihrer Funktion als Mittel zur Symbolisierung von Bezügen funktionieren. Eine konsequente Steigerung dieser Situation ergibt sich in den Verhörsituationen, wo Sprache in letzter Konsequenz als Medium des Psychoterrors eingesetzt wird. Olga werden grausige Geschichten von bestialischen Schwangerschaftstests und -abbrüchen von ihrem Folterer Filinto Müller erzählt. Als Instrument der Machtausübung dient Sprache dem dominanten Gesprächsteilnehmer dazu, sich und andere seiner Potenz zu versichern:

FILINTO [...] Ich bin stark, Olga. Manchmal habe ich fünf oder sechs Frauen in einer Nacht geliebt, manchmal nur drei; ich hatte ziemlich gute ... Muskeln. Ich habe sie noch. Die Frauen haben das nicht ausgehalten, sie konnten so viel Kraft, so viel Macht nicht ertragen. Sie stöhnten sich zu Tode, andere platzten mit aufgequollenem Bauch, wieder eine andere starb an Auszehrung. Sie konnte weder schlafen noch essen, dachte nur noch an mein Bett. (LOHER 1994: 11) 
Filintos Selbstinszenierung als Arbeit am Mythos wird von Olga konterkariert, wobei Loher geschickt selbstreferenziell arbeitet, indem sie mit ironischem Seitenblick auf Seghers und Hermlins Aufsätze sowie Werners Buch über Benario die Autothematisierung von Literatur vornimmt:

OLGA Dann bin ich also der - noch - lebende Beweis dafür, wie weit eine Frau es bringen kann, heutzutage. Einen Mann, ein Kind, eine Laufbahn - vielleicht sogar Berühmtheit. Ich werde zukunftsweisend sein, für alle Mädchen, Frauen, Mütter. [...] Wenn es Biographien geben wird über mich, werde ich darin schön und klug und tapfer und konsequent und mutig sein und den Rücken immer kerzengerade halten. (LOHER 1994: 24)

Als Reaktion auf die Erosion alter Deutungsmuster im Gefängnis benutzt Olga Erzählungen, die jenseits jeglicher Überprüfbarkeit im Mythischen eine sinnstiftende Erklärung ihrer Umstände anstreben. Hans Blumenberg verwies bereits 1979 in seinem Standardwerk Arbeit am Mythos darauf, dass der Mensch aufgrund der als lebensfeindlich empfundenen Wirklichkeit und der daraus resultierenden Erfahrung von Beschränkung und Ohnmacht narrativer Hilfsmittel bedürfe, „um eine Welt zu ertragen und in einer Welt zu leben“, deren Gesetze sich ihm noch nicht erschlossen haben (BLUMENBERG 1979: 59).

Filinto wie Olga liefern in ihren Sprechakten Beispiele für die Arbeit am Mythos, den Olga jedoch wiederum ironisch untergräbt, wenn sie sich dem Status als zukünftige Märtyrerin entziehen will und zu Filintos Nemesis wird. Olga motiviert ihre Gewaltbereitschaft damit, dass sie sich der glorifizierenden Behandlung durch die Nachwelt zu entziehen suche: „OLGA Der einzige Weg, keine Heldin zu sein, keine Märtyrerin, kein Opfer, ist, ich mache mich zur Mitwisserin, Mittäterin. Ich foltere selbst. Foltere jeden, der mir in den Weg kommt.“ (LOHER 1994: 17) Dies trifft auf ihre Behandlung von Filinto genauso zu wie auf politische Gegner. Loher exerziert deshalb die widersprüchlichen Aspekte in der Beziehung zwischen Täter und Opfer vor, die Ambivalenz von Gut und Böse. Olgas Monolog IV ist hier aufschlussreich. Olga beschließt, den Rollentausch $\mathrm{zu}$ probieren, und reflektiert gleichzeitig den Identitätsverlust bzw. die Negation der bisherigen Identität mit allem was dazugehört, besonders ihre Erinnerungen: „OLGA [...] Ich foltere mir mein Hirn tot. [...] Der Ausweg ist die Leere. Das Vergessen. Sich nicht mehr erinnern. Ich erinnere mich an nichts. Was ich jemals wußte, ist fort. [...] Das warst Du, OLGA, sagt Filinto breitbeinig über mir.“ (LOHER 1994: 17) Während Olga bei ihrem ersten Verhör eindeutig in die 
Opferrolle gedrängt wurde, kehrt sie in der Szene „PAS DE DIABLE II“ die Vorzeichen um. Bewusst lässt sich Olga auf den Tanz mit dem Teufel ein und beginnt, Regie zu führen: „OLGA Ich bin eine Verräterin [...] wie Du. [...] Ich will ein Verhör. Ein Verhör bis zum letzten Schrei.“ (LOHER 1994: 18) Olga erklärt den geschmeichelten Filinto zu ihrem Lehrherren: „Ich werde von Dir lernen. Ich werde kein Opfer sein. Ich werde nicht den Schmerz erdulden, ich werde ihn zufügen. Man wird mich fürchten und verachten.“ (LOHER 1994: 18) Der Autosuggestion folgt die Beeinflussung des Bewusstseins von Filinto, der Olga in der Psychologie des Folterns unterweist, nur um am Ende selbst zu ihrem Opfer zu werden. Auf Olgas Frage, „Wie wirst du beginnen?“ (LOHER 1994: 18), offenbart Filinto seine Vorgehensweise im Detail und bemächtigt Olga somit, zum Angriff überzugehen. Auch die Hemmschwelle, psychische und physische Gewalt anzuwenden, entlarvt Filinto als „Konvention“, als eine „scheinheilige Hemmschwelle, gesetzt durch Erziehung. Sie existiert nicht wirklich.“ (LOHER 1994: 19) Olgas Ohnmacht gegenüber Filinto wandelt sich durch das neugewonnene Wissen in Macht über ihn. Olga kann dem Redefluss Filintos entnehmen, dass er einen wunden Punkt hat, wenn es um seine Familie geht: „FILINTO Meine Familie geht dich einen Dreck an. Ich liebe meine Frau. Meine Frau liebt mich, sie liebt und achtet mich, ja, sie ist sogar stolz auf mich, und für meine Kinder bin ich ein Heiliger.“ (LOHER 1994: 19) Filinto erscheint überspitzt defensiv in der Intention, sein Privatleben zu schützen und gerade so verrät er ein traumatisches Ereignis in der Familie. Diesen Umstand nutzt Olga zum Angriff. Sie schlüpft in die Rolle des Peinigers und verkehrt so die Rollen von Täter und Opfer. Sie pervertiert das Gespräch $\mathrm{zu}$ einem Verhör und bohrt sich in Filintos Unterbewusstsein vor, bis sie seine unterdrückte Erinnerung zum Vorschein gebracht hat: „FILINTO Ich wußte nicht, daß ich zuhause war. [...] Ja, ja, ich habe sie [meine Frau] verletzt. [...] ich erkannte sie nicht.“ (LOHER 1994: 20) Olga exerziert in dieser Szene die Paradoxie des Verhöres vor, die sie im folgenden Monolog auf theoretischer Ebene reflektiert:

Der Widerspruch in der Folter, die Folter ist: Du sollst Nichts wissen, dich erinnern, daß Nichts war. Vergessen was war. Sie sollen dir glauben, daß du Nichts weißt, aber du weißt alles, mußt dir dein Gedächtnis, Erinnerung, Wissen bewahren um zu bleiben du selber. Gleichzeitig vergessen und die absolute Erinnerung bewahren. Den Folterer über deine UltraErinnerung, dein HyperGedächtnis hinwegtäuschen, ihm in jedem Moment die geistlose NurGegenwart, die leere Vergangenheit VORSPIELEN. (LOHER 1994: 21)

Das Rollenspiel meint in diesem Verständnis nicht nur Öffnung im Vergleich mit Filinto, sondern argumentative Auseinandersetzung mit seiner Sicht- und 
Ludewig, A. - Dea Lohers Olgas Raum

Vorgehensweise. Dieser Prozess verlangt Metakognition, im Sinne einer kontinuierlichen Selbstbeobachtung und Adaption. So sind auch Olgas letzte Worte zu verstehen, "leicht lebe ich fort" (LOHER 1994: 34), als losgelöste Form, die in der Gegenwart weiterlebt, da Erinnerung, Gedächtnis und Geschichte diverse Geschichten ihre Person weiter- und fortschreiben, egal ob als Opfer oder Täter, Hauptsache als Handelnde.

Den Raum, die Zelle, die man ihr lässt, sprengt Olga mit ihren Worten, d. h., die drei Dimensionen, die den Raum begrenzen, transzendiert sie durch die vierte Dimension: die Zeit, die sie in ihrem Kopf Revue passieren lässt. Meist ist es dämmrig, dunkel, doch nie düster, denn die Farben und Lichter, die wechselnden Stimmungen, die diese Einsiedelei dennoch erfüllen, speisen sich aus Olgas Erinnerung. Sie beleben den Raum, bis zum Angesicht des Todes. Erst am Schluss sieht sich Olga, allein in einem leeren Raum, der Möglichkeit weiterzusprechen beraubt. Der Raum, vormals voller Erinnerungen, Erfahrungen, Erlebnisse, die maßgeblich an der Ausbildung ihrer Identität beteiligt gewesen waren, erscheint nun leer und hat jede Magie verloren, er ist nur noch Kammer, Todeskammer.

OLGA Ich bin allein.

Das ist mein - Raum.

Vernichtungsanstalt.

Ich bin angekommen.

Bernburg.

Ich nenne den Ort beim Namen.

Ich bin Olga. Ich bin allein.

Das ist mein leerer Raum.

Eine - Kammer. (LOHER 1994: 34)

Die Propaganda der Nazi-Zeit, die von Lebensraum, Volk ohne Raum und der Erweiterung des Lebensraums im Osten sprach, und die Ausführung dieser Verheißungen von Menschen, die der Idee vom deutschen Herrenvolk nicht abgeneigt waren, lassen Olga keinen Raum mehr, wie sie generell Andersdenkenden keinen Freiraum einräumen wollten. Im letzten beklemmenden monologischen Bekenntnis von Olga scheinen ihre Widersacher erfolgreich, indem man ihr den letzten Raum nimmt. Doch Dea Loher verkehrt diese Situation zu einem Sinnbild für Freiheit. Sie bekennt in einem Interview: „Theater ist der Raum der Sprache [...] ein je größeres Eigenleben die Sprache entwickelt, je verdichteter und konzentrierter sie ist, desto größer ist der Spielraum für die Phantasie, den sie freisetzt.“ (LOHER in ŠTĚDrOŇ 2004: 269). Diese Methode kulminiert nach einer wortgewaltigen Aufführung im jähen Verstummen der 
Sprache. Olga verlässt den Bühnenraum und überlässt den Zuschauern Olgas leeren Raum. Diesen füllt nun jeder mit seinen eigenen Gedanken, einer neuen Konstruktion, einem neuen Ort. Olgas Raum wird zu einem neuen Raum mit dem Potenzial für eine umfassende Revidierung. In der Trostlosigkeit des letzten Bildes steckt somit die positive Botschaft der Theaterautorin, die das Auditorium zur Erweiterung der Bühne macht und jeden Einzelnen auf das eigene Potential verweist. Daraus entsteht die Option von Heteroglossie, d. h., eine Vielstimmigkeit in der Gedankenarbeit der Rezipienten. In der Aufführungspraxis wird diese Einbeziehung der Zuschauer, die in der Struktur von Olgas Raum als Appellfunktion integriert ist, vielfältig umgesetzt: Der Rezipient (Leser / Zuschauer) ist bei Dea Lohers Textproduktion mitgedacht. Dieser den Bühnenraum erweiternde, transzendierende Vorgang, der inhaltlich vom Text konditioniert ist, erlaubt es dem Rezipienten, sich selbst mitzudenken. Dieser Akt der Involviertheit wird begünstigt durch Olgas Fehlbarkeit, die ihre menschlichen statt mythisch-überhöhten Züge betont.

Dea Loher thematisiert hier das Wesen der Menschen selbst, indem Olgas Rolle dieses anthropologische Dilemma kommentiert: „Am Ende stirbt der Mensch an seiner Wahrheit. Weil er wahr ist, weil er war, weil er ist, Mensch.“ (LOHER 1994: 21) Diesem Umstand trägt der aus Magdeburg stammende Regisseur und Castorf-Schüler Andreas Kriegenburg in seiner Inszenierung des Stückes Rechnung durch sein „Interesse an der Austauschbarkeit von Täter und Opfer, seine szenischen Zuspitzungen in diesem Punkt - aus dem Opfer wird eine Täterin -, wie sein Versuch, die konkreten politischen Bedingungen $\mathrm{zu}$ entzeitlichen, durch die Entindividualisierung der drei Frauen in Kostümen, Haarfrisuren oder durch choreographierte Bewegungen" (BöRGERDING 2007: 337). Loher hinterfragt mit ihrem Text Opfer- und Täter-Kategorie ohne Schuldzuweisungen oder Verurteilungen, denn es geht ihr - wie Loher an anderer Stelle betonte - nicht darum „unmittelbar etwas anzuklagen“ (LOHER in GROSS / KHUON 1998: 225). Was LÖSCHNER für Olga konstatiert - „Die Benario in Dea Lohers Stück ist keine Heldin, sie taugt nicht zur Märtyrerin“ (1997: 72) - gilt genauso für die anderen Akteure auf der Bühne wie für die Personen vor der Bühne. Loher will dieses Rollenspiel als geistige Projektion weitertreiben, in dem sie die Gefängniszelle in Südamerika zu einem Allgemeinort macht. Der Ort wird transparent für andere Zeiten, Systeme, Akteure. In diesem vierten Raum, dem imaginierten, müssen stalinistische Gulags genauso mitgedacht werden wie Guantanamo Bay. So wird der Opferstatus von 
Olga Benario arbiträr und ihre Täterschaft zentral, denn jeder Mensch ist eben nur das, ein Mensch. Loher kommentiert die Fehlbarkeit der Figuren in Olgas Raum wie folgt: „Dass die Figuren in den Stücken stellvertretend für mich als Zuschauer kämpfen und leiden und manchmal auch unterliegen, finde ich sehr tröstlich.“ (LOHER in ŠTĚDROŇ 2004: 279). Olga Benario wird so - nach all der Überhöhung der bisherigen Geschichtsschreibung - wieder menschlich.

\section{Schlusswort}

In Dea Lohers Olgas Raum geht es um die Offenlegung der Konstruktionsmechanismen von Geschichte und Geschichten. Als Zuschauer wird man nicht nur auf diese Logik hingewiesen, sondern selbst in die Verantwortung genommen. Diese aktive Rezeption wird begünstigt durch die Kombination von Monolog- und Dialogpassagen, die ihrerseits sowohl den Perspektivenwechsel als auch eine Verkomplizierung der Geschichte(n) nahelegen. Schlussendlich ist der Zuschauer mit dem leeren Raum, den Olga verlässt, alleingelassen und muss in der entstehenden Stille ein Potenzial sehen, die Geschichte(n) weiterzuspinnen. Jede Hoffnung auf ein Happy Ending ist verblasst und die Flucht in einen Mythos wenig attraktiv, nachdem man mit der Einsicht konfrontiert wurde, dass tragische Fehlbarkeit zutiefst menschlich und nicht mythisch ist.

\section{Literaturverzeichnis}

BLUMENBERG, Hans. Arbeit am Mythos. Frankfurt a.M.: Suhrkamp, 1979.

BÖRGERDING, Michael: „I'm just blue'-Der Regisseur Andreas Kriegenburg und seine Auseinandersetzung mit den Texten der Dramatikerin Dea Loher“, Monatshefte, Vol. 99, Number 3, Fall, 2007. S.333-345.

COHEN, Robert. Exil der frechen Frauen. Berlin: Rotbuch, 2009.

FISCHBORN, Gottfried. Stückeschreiben. Claus Hammel, Heiner Müller, Armin Stolper. Berlin: Akademie-Verlag, 1981.

FuKuYAma, Francis. "The End of History?”, The National Interest. Summer, 1989. S.3-18.

HAAS, Birgit. "Dea Loher: Vorstellung", Monatshefte, Vol. 99, Number 3, Fall 2007; S.269279.

HermLin, Stephan. „Olga Benario“, in: ders., Die erste Reihe. Berlin: Verlag Neues Leben, 1951. S.64ff.

JUDT, Tony. „Vom Neuschreiben der Geschichte. Erinnerungspolitik nach 1945 und 1989“, in: Transit. Europäische Revue. Heft 15, Herbst, 1998. Wien: Verlag Neue Kritik. S.3-11. 
LOHER, Dea. „Rede zu Verleihung des Gerrit-Engelke-Preises“, in: Dea Loher und das Schauspiel Hannover. Hrsg. Jens Groß; Ulrich Khuon, Hannover, 1998. S.224-229.

LOHER, Dea. Olgas Raum, Frankfurt a.M.: Verlag der Autoren, 1994.

LÖSCHNER, Sascha. „Verletzte Sprache“, in: Stückwerk. Deutschsprachige Dramatik der 90er Jahre. Berlin, 1997. S. 71-73.

Miguel, Júlia Mara Moscardini. „Dea Loher e a Arte de Costurar Histórias Dramaturgicamente". Pandaemonium Germanicum, São Paulo, v. 19, n. 27, 20016. S.27-47.

MORAIS, Fernando. Olga. Das Leben einer mutigen Frau. Köln: Volksblatt-Verlag, 1989.

PANITZ, Eberhard. Jorge Amados Einmischung. Ein Schriftstellerleben. Berlin: Spotless-Verlag, 1998.

SEGHERS, Anna. „Olga Benario-Prestes [1951]“, in: dies. Über Kunstwerk und Wirklichkeit. Bd. III. Herausgegeben von der Deutschen Akademie der Wissenschaften zu Berlin. Bearbeitet und eingeleitet von Sigrid Bock. Berlin: Akademie-Verlag, 1971, S. 158-61.

ŠTĚDrOŇ, Petr. „Dea Loher - Die Elf glücklosen Stücke“, https://digilib.phil.muni.cz/bitstream/handle/11222.digilib/106049/1 BrunnerBeitratge GermanistikNordistik_18-2004-1_15.pdf?sequence=1, 2004, S.265-280.

Toledo, Sergio. One Man's War (Film), UK 1990.

WERNER, Ruth. Olga Benario - die Geschichte eines tapferen Lebens. Berlin: Neues Leben, 1961. 